\title{
Tensions, diplomatie et accommodements dans un espace partagé : la France et l'Angleterre sur la côte ouest de Terre-Neuve II (1842-1870)
}

\section{Nicolas Landry}

In an article previously published in this journal, it was stated that maintaining order in Newfoundland's disputed shared maritime space was a major challenge in the first half of the $19^{\text {th }}$ century. This follow-up article attempts to show that, as of 1840, diplomats working in metropolitan areas and Franco-British frigate officers patrolling the coast of Newfoundland were preoccupied with two closely related issues in terms of maintaining order. While they were expressing their respective colonial ambitions elsewhere on the planet, France and England advocated for a gradual diplomatic rapprochement that would lead to two important agreements, namely the 1854 Alliance during the Crimean War and the Entente Cordiale of 1904. In a context such as this, the quest for compromise became a fundamental responsibility of naval officers in both countries. Like diplomats at the bargaining table, they had to be able to blow hot and cold in order to assert themselves but also to exercise restraint and judgment. Diverting from this course of action could have had repercussions in the back rooms of Europe and jeopardized the Franco-British process of rapprochement.

La première partie de cette recherche, publiée dans un article précédent, nous amène à conclure que le maintien de l'ordre dans l'espace partagé francobritannique de la côte ouest de Terre-Neuve durant la première moitié du $19^{\mathrm{e}}$ siècle se caractérise par une inébranlable volonté de compromis entre les autorités des deux nations ${ }^{1}$. L'on est cependant en droit de se demander si cette zone d'interaction

\footnotetext{
1 «Tensions, diplomatie et accommodements dans un espace partagé entre Saint-Pierre et Miquelon et Terre-Neuve, 1816-1842 », The Northern Mariner/Le marin du Nord 27:2 (April 2017), 143-162.
}

The Northern Mariner / Le marin du nord, XVIII, No. 4 (Autumn 2018), 347-62 
internationale était alors unique. Comment mieux situer cette problématique dans l'ensemble des relations franco-britanniques, incluant les États-Unis? Il est permis de croire que non si l'on s'en remet à l'exemple de la Manche qui, selon Morieux, représente aussi un "lieu commun » pour les pêcheurs et autre usagers francobritanniques à la même époque. Cet auteur parle ainsi d'une «étude des interactions » que l'on peut, à notre avis, appliquer aux réalités de la côte de Terre-Neuve².

Notre découpage chronologique s'inspire des travaux de Kurt Korneski, démontrant qu'une certaine escalade des tensions se manifeste sur la côte ouest de Terre-Neuve entre 1850 et l'entente cordiale de $1904^{3}$. Le présent texte tente donc de déterminer s'il existe effectivement un climat de tension plus perceptible dans les relations franco-britanniques après 1850 . À titre d'exemple concret de tensions survenant sur la côte de Terre-Neuve, mentionnons deux pillages d'épaves au détriment d'un navire de Saint-Pierre et Miquelon. D'abord, en 1826, survient le naufrage et le pillage d'un navire français à Ship Cove par des habitants de Fortune. Également, en 1856-57, un sérieux test des relations franco-britanniques survient dans la région, lors du pillage d'une goélette de Saint-Pierre, soit la Marie Élisabeth. Cet événement survient sur la « pointe de Rose Blanche» de TerreNeuve ${ }^{4}$.

\section{Une historiographie renouvelée}

Cette recherche se comprend mieux si on l'inscrit dans l'histoire des deux empires coloniaux de l'époque que sont ceux de la France et de l'Angleterre. Les États-Unis, pour leur part, occupent moins de place dans le conflit de Terre-Neuve que dans celui des Maritimes. Si, dans le cas de Terre-Neuve, il s'agit avant tout de tensions émanant de droits d'accès aux fonds de pêche et à des espaces de séchage, la volonté d'y empêcher un peuplement permanent ajoute un facteur de tension n'existant pas sur le littoral des provinces maritimes. Dans cet autre espace contesté, la rivalité n'implique que les gouvernements américains et anglais alors qu'à Terre-Neuve, les trois nations se disputent l'accès à la ressource. Notre recherche se limite donc au seul espace contesté qu'est Terre-Neuve et se penche en exclusivité sur la rivalité franco-britannique.

L'historiographie des droits de pêche sur les côtes de Terre-Neuve semble connaître son âge d'or à compter de la fin du $19^{\mathrm{e}}$ siècle, pour se prolonger au début du siècle suivant ${ }^{5}$. Ce thème de recherche reprend de la vigueur avec la publication

2 Renaud Morieux, « La Manche au XVIII ${ }^{e}$ siècle. La construction d'une frontière franco-anglaise », Annales historiques de la Révolution française 343, janvier-mars 2006, 167-174.

3 Kurt Korneski, « Development and Diplomacy: The Lobster Controversy on Newfoundland's French Shore, 1890-1904 », The International History Review 36:1, 2014, 45-69.

4 Saint-Pierre. Témoignages des membres d'équipage de la Marie Élisabeth devant le commissaire de l'inscription maritime de Saint-Pierre et Miquelon, 15 novembre 1856. BAC, MG1-C11C, vol. 7b, bobine F-505, 4 pages. Rose Blanche est une petite communauté côtière située sur la côte sud-ouest de Terre-Neuve.

5 À ce sujet, voir Jean-François Brière, «Pêche et politique à Terre-Neuve au XVIII siècle; la France véritable gagnante du traité d'Utrecht? », Canadian Historical Review 64:2,(1983), 170, note 
de Frederic F. Thompson, suivi des nombreux travaux de J.K. Hiller et de Peter Neary $^{6}$. Ces deux chercheurs contribuèrent certes à renouveler nos connaissances sur cette facette de l'historiographie du Canada atlantique. Si, depuis un certain temps, la question du French Shore semble susciter moins d'intérêt chez les chercheurs francophones, un souffle nouveau se manifeste du côté anglophone selon les travaux de Brian J. Payne, Thomas Blake Earle et Kurt Korneski. Ces auteurs partagent deux grandes thèses.

En premier lieu, ils s'entendent pour dire que les législations et les traités ne répondent pas aux réalités prévalant dans l'environnement de l'Atlantique nord et chez les populations des littoraux du Canada atlantique. Par exemple, Payne utilise le terme « territorialité environnementale » qui, selon lui, implique le respect d'un code de conduite créé et mis en pratique par les pêcheurs canadiens et américains ${ }^{7}$. Selon lui, ce système fonctionne si l'on ne tente pas de l'articuler dans les contraintes diplomatiques imposées conjointement par les autorités britanniques, américaines et canadiennes.

En deuxième lieu, les frontières artificielles découlant des traités sont incompatibles avec le code de conduite pratiqué par les habitants des littoraux et les pêcheurs des trois nations. Ce sont donc ces travailleurs maritimes qui insistent, même à l'encontre de leur propre gouvernement, pour exercer un contrôle sur les ressources locales. Dans ce cas-ci, la boëtte et le hareng. Ces réalités ne peuvent pas être ignorées, du moins jusqu'au début du $20^{\mathrm{e}}$ siècle alors que se manifeste les innovations technologiques telles que les seines en bourse et les chaluts. Ainsi, jusqu'à ce moment-là, les navires étrangers doivent forcément pénétrer dans les eaux côtières pour se procurer de la boëtte en échange de marchandises de toutes sortes. Donc, pour les pêcheurs canadiens et terre-neuviens, le commerce américain et français représente une alternative viable au monopole des marchands britanniques ${ }^{8}$.

Thomas Blake Earle, lui, est d'avis que les traités statisques ne peuvent pas réguler la dynamique environnementale ${ }^{9}$. Il explique ainsi quelle logique environnementale se manifeste à compter de 1800; les populations de morue sont en déclin et le maquereau devient une alternative viable. Les pêcheurs, eux, s'adaptent et doivent modifier leurs façons de faire. Mais, au contraire, les pratiques administratives

\section{7.}

6 F.F. Thompson, The French Shore Problem in Newfoundland. An Imperial Study, (Toronto: University of Toronto Press, 1961). Cet ouvrage de facture classique s'intéresse aux actions diplomatiques françaises, anglaises et terre-neuviennes. Pour Peter Neary, voir en premier The French and American Shore questions as factors in Newfoundland History, (Toronto: University of Toronto Press, 1961). J.K. Hiller, « The Newfoundland Fisheries Issue in Anglo-French Treaties, 1713-1904 », The Journal of Imperial and Commonwealth History 24:1 (January 1996), 1-23.

7 Brian J. Payne, Fishing the Borderless Sea. Environmental Territorialism in the North Atlantic, 1818-1910,( Lansing, Michigan: Michigan State University Press), 2010, 128.

8 Ibid., 271.

9 Thomas Blake Earle, «Transatlantic Diplomacy, North Atlantic Environments, and the Fisheries Dispute of 1852 », Environmental History, https://doi.org/10.1093/envhis/emy006. Published April 2, 2018, 31 pages. American Society for Environmental History, 1-2. 
conduisant les pêcheries ne semblent pas en mesure de le faire. Quant à Korneski, il estime que des tensions peuvent survenir où des puissances étrangères exercent des pouvoirs que leur confèrent les traités. C'est ainsi que la côte ouest de TerreNeuve représente un espace frontière, où se côtoient des intérêts et des enjeux nationaux et impériaux. N'empêche que c'est sur ce même territoire que se forgent des aspects distinctifs d'arrangement économiques, culturels, politiques et sociaux ${ }^{10}$.

À l'image de ce que nous soulevons dans notre article précédent, Korneski pense lui aussi que depuis 1815 , des échanges commerciaux existent entre les résidents de la côte sud-ouest de Terre-Neuve et les Français, qu'ils soient de la métropole ou de Saint-Pierre et Miquelon. Il pouvait difficilement en être autrement puisque les pêcheurs métropolitains utilisent ce territoire, incluant l'archipel, à titre de base d'opération pour leur pêche sur les bancs. Ainsi, une bonne partie de la population de Fortune Bay et des environs tire une part importante de sa subsistance de ce trafic. Il ne faut donc pas se surprendre que cette population s'objecte à toute intervention gouvernementale risquant de perturber ce commerce.

\section{Objectifs de recherche}

C'est donc dans ce contexte historiographique stimulant que souhaite s'inscrire la deuxième étape de notre recherche sur la cohabitation des autorités francobritanniques sur le French Shore de Terre-Neuve. Les deux principales sources archivistiques pour cette étude sont les fonds coloniaux français $\mathrm{C} 11^{\mathrm{C}}, \mathrm{C} 11^{\mathrm{F}}$ et $\mathrm{G}^{1}$, accessibles dans la base de données Archivianet de Bibliothèque et Archives Canada, de même que celui du Colonial Office, série 194. L'accès à ce dernier passe par la consultation de l'inventaire dressé par le professeur Olaf Janzen ${ }^{11}$. À partir de ces fonds, nous tentons de faire ressortir les tentatives des fonctionnaires et des officiers de Marine de France et d'Angleterre, pour modérer les ardeurs des pêcheurs, maintenir l'ordre dans cet espace colonial partagé qu'est le French Shore (côte sud-ouest de Terre-Neuve) et affirmer les droits de pêche des deux nations.

Mais pourquoi préconiser cette politique de compromis et d'apaisement? D'abord, qu'en est-il sur la scène internationale en termes de relations bilatérales entre la France et l'Angleterre après la Restauration de 1815? Un survol des événements survenant durant la période à l'étude permet de constater que les deux empires avaient tendance à souffler le chaud et le froid. D'abord, dans la foulée de la Restauration, la France tient à rassurer l'Angleterre que le « rétablissement du nouvel Empire français $»^{12}$ ne cache aucune intention belliqueuse. Pour arriver à ses fins, entre 1827 et 1850, la France entreprend quatre expéditions dans le Pacifique

10 Kurt Korneski, Conflicted Colony. Critical Episodes in Nineteenth-Century Newfoundland and Labrador (Montréal \& Kingston) McGill-Queen's University Press), 2016, 7-8.

$11 \mathrm{http} / / / \mathrm{www} 2 . \mathrm{swgc} . \mathrm{mun}$.

12 Quentin Courcelas, «Alexandre Walewski, un acteur de la diplomatie du Second Empire : l'ambassade de Londres (1851-1855) », La Fondation Napoléon / Napoleonica. La Revue, $\mathrm{n}^{\circ}$ 30, 2017,61 . 
afin d'assoir son autorité au Tonga, à Fiji et en Nouvelle-Calédonie, qu'elle finit par annexer en $1853^{13}$. Rappelons qu'elle s'empare de l'Algérie en 1830, ce qui ne manque pas d'inquiéter les politiciens britanniques. L'on ne se surprend pas que la stratégie française consiste alors à réagir aux avancées britanniques en Australie et en Nouvelle-Zélande ${ }^{14}$.

Pour revenir à la politique de compromis préconisée à Terre-Neuve, émettons quelques hypothèses à cet effet : les représentants franco-britanniques à TerreNeuve n'ont pas les moyens nécessaires permettant d'inculper et de punir les coupables, chacune des parties craint de provoquer une riposte à une action jugée trop radicale, une étincelle de violence dans cet espace colonial partagé risque d'avoir des répercussions ailleurs dans l'empire et, finalement, la possibilité que l'éloignement des métropoles fasse en sorte que l'application des traités puisse se pratiquer avec un certain laxisme accommodant. Nous osons croire que ce texte permet d'apporter des réponses à ces questions ${ }^{15}$. Mais, en 1851, ces entreprises coloniales n'empêchent pas Louis-Napoléon Bonaparte de penser qu'une « alliance étroite avec le Royaume-Uni »s'avère « indispensable au progrès de la civilisation $»^{16}$. D'ailleurs, la philosophie diplomatique de son ambassadeur à Londres Alexandre Walewski sied bien à celle mise de l'avant par les officiers de frégates franco-britanniques à Terre-Neuve, soit de « savoir discerner les limites $\mathrm{du}$ possible et circonscrire ses efforts» ${ }^{17}$. Ce rapprochement franco-britannique conduisant à la future entente cordiale se concrétise en 1854 alors que les deux pays forment une alliance lors de la Guerre de Crimée.

Dans ce contexte, il est utile de revenir sur les efforts diplomatiques consacrés spécifiquement à la question des droits de pêche à Terre-Neuve. En 1839, la convention franco-britannique s'intéresse à la délimitation des pêcheries ${ }^{18}$. Le 25 février 1842, la France, elle, émet une ordonnance royale entérinant une loi sur la pêche à la morue datant de $1841^{19}$. Deux ans plus tard, soit le 24 mai 1843 , survient le règlement international sur les pêcheries entre la France et l'Angleterre. Toutefois, même si les relations diplomatiques sont plutôt harmonieuses entre 1843 et 1845, les choses s'avèrent moins faciles à Terre-Neuve ${ }^{20}$. En effet, même si les deux métropoles en viennent à un accord le 23 janvier 1857 , ce dernier est

13 Bronwen Douglas, «Affect, Agency, and Ambiguous Praxis: French Expeditions in the Pacific Islands, 1827-50 », Journal of Colonialism and Colonial History 18:1 (Spring 2017), 1.

14 Ibid., 9.

15 Korneski aborde cette problématique en analysant certains épisodes de tension survenus sur la côte sud-ouest de Terre-Neuve tout au long du 19e siècle. Korneski, Conflicted Colony.

16 Courcelas, 61.

17 Ibid.,78.

18 « Texte du droit commercial maritime », 2 août 1839, vol. 9, p. 292. Dans Notions du droit maritime. https://gallica.bnf.fr/ark.

19 Annales maritimes et coloniales, vol. 32, p. 126. Imprimerie royale, 1847, 3e série, 1842 à 1847.

20 À l'époque, les commissaires de Montaignac et de Gobineau estiment à 3000 la population anglaise établie sur la côte française de Terre-Neuve. R. Rompkey, Les Français à Terre-Neuve : un lieu mythique, une culture fantôme, (Bordeaux: Presses universitaires de Bordeaux, 2009), 280. 
rejeté par le Parlement de Terre-Neuve ${ }^{21}$. Cet échec est suivi par les travaux d'une commission mixte entre mai et août 1859.

En guise de mise en contexte de la cohabitation franco-britannique autour de l'archipel et sur la côte sud-ouest de Terre-Neuve, il est utile de s'initier à certains aspects environnementaux et sociaux de Saint-Pierre. La colonie de Saint-Pierre et Miquelon a pour chef-lieu l'île Saint-Pierre, qui abrite la capitale administrative et ses fonctionnaires. De manière plus précise, ce territoire fait face à la côte méridionale de Terre-Neuve au $46^{\mathrm{e}}$ degré de latitude septentrionale et au $58^{\mathrm{e}}$ degré de longitude du méridien de Paris. Vers 1819, il y a à Saint-Pierre 55 cabanes d'habitants avec une population d'environ 200 personnes ${ }^{22}$. C'est ainsi que la population passe de 600 personnes en 1816 à 4750 en 1870 . Et ce, malgré les grands incendies de 1844,1865 et 1867 . En ce qui a trait au nombre de goélettes armant à partir de l'archipel, leur nombre passe de 20 en 1825 à 284 en $1903^{23}$.

\section{Inscrire l'histoire coloniale dans l'histoire atlantique}

Selon Allain Cabantous, parler d'histoire coloniale est aussi parler d'histoire « des terres colonisées », qui représenterait une «fraction de l'histoire atlantique ». Il pense également qu'il ne faille pas limiter l'approche de l'histoire atlantique au premier tiers du $19^{\text {e }}$ siècle. Ainsi, les échanges et les migrations augmentent substantiellement après $1840^{24}$. D'ailleurs, Jean-François Klein soutient que la période 1789-1879 a jusqu'à maintenant été « très peu travaillée par les historiens du moment colonial $»^{25}$. À un autre égard, Gérard Le Bouëdec qualifie le $19^{\mathrm{e}}$ siècle comme étant «celui de l'intégration des mondes littoraux dans la communauté nationale » française. Bref, il s'agit là de la « rencontre entre ceux du rivage et ceux de l'intérieur ». C'est aussi l'époque où la «grande pêche de Terre-Neuve retrouve son dynamisme $»^{26}$.

L'on se rappelle qu'en 1778, les Anglais s'emparent de l'archipel de Saint-Pierre et Miquelon pour la première fois et détruisent à peu près toutes ses infrastructures. Le Traité de Versailles du 8 septembre 1783, mettant fin à la Guerre d'Indépendance américaine, permet à la France de reprendre l'archipel. En contrepartie, la France

21 À ce sujet, le lecteur peut aussi se référer à la Convention relative à la pêche sur les côtes de l'île de Terre-Neuve et sur les côtes avoisinantes. Londres, 14 janvier 1857. Recueil Manuel et pratique de traités et Convention, 1857-1869. https://books.google.ca.

22 Aperçu sur la statistique des îles Saint-Pierre et Miquelon, 9 novembre 1819. BAC, MG1-G1, vol. 463, bobine F-767, fol. 167-181v.

23 Marc Pabois, «L'archipel de Saint-Pierre et Miquelon », In Situ. Revue des patrimoines, 3/2003. Les horizons de l'inventaire. Revues_org/2064 :DOI :10.4000/. André-Louis Sanguin, «Un archipel balloté entre deux puissances coloniales », Norois, ${ }^{\circ}$ 110, avril-juin 1981, 136-154.

24 Alain Cabantous, « Résistance de principe ou lucidité intellectuelle? Les historiens français et l'histoire atlantique », Revue historique, 2012/3, n 663, 709, 714.

25 Jean-François Klein, « Un chaînon manquant impérial? Les Monneron, une fratrie d'armateurs au tournant du monde », dans Jean-François Klein et Bruno Marnot, Les Européens dans les ports en situation coloniale $X V I^{e}-X X^{e}$ siècle, (Rennes: Presses universitaires de Rennes, 2014), 43.

26 Gérard Le Bouëdec, «Le temps des crises dans les sociétés littorales (XVI ${ }^{\mathrm{e}}-\mathrm{XIX}^{\mathrm{e}}$ siècle) », Annales de Bretagne et des Pays de l'Ouest, 117-3/2010, 13-24. 
renonce à son droit de pêche depuis le Cap Bonavista jusqu'au Cap Saint-Jean. Mais les troubles découlant de la Révolution française et des guerres francobritanniques, se poursuivent de manière pratiquement ininterrompue jusqu'en 1815. La deuxième conquête anglaise de l'archipel survient en 1793, impliquant encore une fois la déportation de la population mais sans, cette fois, une destruction systématique des infrastructures. Quoique le Traité d'Amiens du 27 mars 1802 rétablisse le statut quo d'avant 1793, la France ne réoccupe définitivement l'archipel qu'après le Traité de Paris du 30 mai 1814. Tel que précisé dans notre article précédent (la première partie de cette recherche), la période 1816-1842 est marquée par une alternance de périodes d'apaisement et de tension. Mais qu'en est-il par la suite? Tout indique qu'un scénario à peu près semblable s'impose aux deux nations à compter de 1841 .

\section{Reprise des tensions 1841-1848}

En effet, les tensions diplomatiques sur les fonds de pêche semblent connaître une recrudescence durant les années 1840. Ainsi, dès avril 1843, le ministre de la Marine Albin Roussin alerte son collègue des Affaires étrangères qu'un vaisseau de guerre anglais a défendu à des pêcheurs français de fréquenter le détroit de Belle Isle en $1841^{27}$. Comme réplique, l'amiral Victor Guy Duperré ${ }^{28}$ suggère de rappeler aux Anglais les «droits » de la France et sa «tolérance des Anglais établis sur sa rive terre-neuvienne $»^{29}$. De son côté, le commandant de Saint-Pierre et Miquelon, comme d'autres avant lui, déplore les effets néfastes de la présence britannique à Saint-Georges. Il estime cette population à 2000 personnes qui se sont aussi installées à Cod Roy, où ils pêchent surtout le saumon, le hareng et la morue. Bref, tous s'entendent sur l'urgence que l'ambassadeur français à Londres dénonce l'occupation anglaise des « baies terre-neuviennes françaises ${ }^{30}$.

27 Rappelons que la convention de 1818 défend aux Américains de pêcher à moins de trois milles des côtes canadiennes. L'on peut penser que les Britanniques appliquent les clauses des traités de manière plus agressive qu'envers les Français à Terre-Neuve et ce, durant les années 1820 à 1840 . Il y a en effet des centaines de navires américains appréhendés. Earl, 203.

28 Victor Guy Duperré est officier de Marine, Amiral de France en 1830, Pair de France et ministre de la Marine et des Colonies à trois occasions entre 1834 et 1843. http://fr.wikipedia.org/ wiki/Guy_Victor_Duperre\%.

29 Albin Roussin, ministre, à son collègue des Affaires étrangères, 12 avril 1843. BAC, MG1C11C, vol. 7b, bobine F-505, 1. Rappelons qu'entre 1839 et 1851, 27 navires américains sont saisis à l'intérieur des eaux dites canadiennes, soit les provinces maritimes actuelles. Pour les habitants des côtes canadiennes, même si les traités donnent accès aux eaux côtières, la pêche de la boëtte par des navires étrangers est considérée comme de la contrebande. Payne, 26.

30 Roussin, ministre, à son collègue des Affaires étrangères, 12 avril 1843. BAC, MG1-C11C, vol. 7b, bobine F505, 1-2. À l'origine, selon lui, cette population se compose de « quelques sauvages venus de l'Île Royale », auxquels les Anglais accordent la permission de s'installer. Ils sont ensuite rejoints par quelques familles franco-britanniques. En 1813, la population atteint à peine 200 personnes. En 1843, cette population d'environ 2000 personnes compte 400 amérindiens (20\% du total) et quelques Canadiens à côté des Anglais et des Français. À Cod Roy, des familles anglaises se sont établies à la « petite et à la grande rivière ». 
Quoique les clauses du dernier traité ne reconnaissent pas le droit aux Français de pêcher dans les parages du détroit de Belle Isle, il demeure possible d'invoquer le principe de réciprocité de la tolérance que les Français appliquent eux-mêmes envers la population anglaise établie dans les havres pourtant réservés à la pêche française. Pour éviter tout prétexte de heurt entre les pêcheurs franco-britanniques, les patrouilles françaises de la station de Terre-Neuve tentent régulièrement de dissuader les pêcheurs français de fréquenter cette section de la côte, mais sans succès apparent. Il ne faut pas oublier qu'en 1835, l'objection se manifestant sur la côte sud de Terre-Neuve ne s'adresse pas tant contre la présence française que contre l'usage croissant de très grands filets visant à pêcher la boëtte et le hareng ${ }^{31}$.

Pour bien saisir le contexte changeant dans lequel s'inscrivent les événements de la deuxième moitié du $19^{\text {e }}$ siècle à Terre-Neuve, il est essentiel de faire une mise au point historiographique sur les mutations se déroulant dans les pêcheries françaises à l'époque. D'abord, selon Nathalie Meyer-Sablé, l'année 1830 marque les débuts de l'ère industrielle, entre autres avec l'apparition des « usines le long du littoral breton », accompagné d'un certain « bouleversement du fonctionnement de l'économie des populations littorales » ${ }^{32}$. Mais Marc Pavé place plutôt «autour de 1850 l'industrialisation » des pêcheries. Il est toutefois d'avis que 1840 marque surtout la reprise des débarquements de morue suivant la longue interruption provoquée par les guerres de la Révolution et de l'Empire ${ }^{33}$.

Selon Jean-Marie Auffrey, la grande pêche pratiquée au milieu du $19^{\mathrm{e}}$ siècle est surtout celle qualifiée de pêche sédentaire dans les havres du nord de TerreNeuve, à partir desquels on part pêcher sur de petites embarcations, et où des installations durables permettent de saler et de sécher le poisson. Pendant ce temps, plus au sud, en pleine mer, sur les grands bancs de Terre-Neuve, plusieurs navires malouins pratiquent la pêche à la morue verte avec des lignes de fond. Saint-Pierre et Miquelon sert alors de base arrière ${ }^{34}$.

Les tensions diplomatiques évoquées plus haut montent d'un cran en 1843, alors que les Anglais saisissent trois goélettes françaises à la baie du Désespoir, surprises à charger du bois de chauffage ${ }^{35}$. Elles sont cependant promptement rendues par le gouverneur de Terre-Neuve. Néanmoins, à l'approche de la prochaine saison de pêche 1844, d'autres décisions du gouvernement anglais inquiètent les autorités

31 Earl, 26.

32 Nathalie Meyer-Sablé, Familles des Marins-pêcheurs et évolution des pêches. Littoral Morbihannais 1830-1920 (Paris: L'Harmattan, 2005), 9.

33 Marc Pavé, La pêche côtière en France (1715-1850). Approche sociale et environnementale (Paris: L'Harmattan, 2013), 58.

34 Jean-Michel Auffray, « Entre grande pêche et commerce. Les reconversions des armements bretons sous le Second Empire », Annales de Bretagne et des Pays de l'Ouest, 117-4/2010, 167.

35 Saint-Pierre et Miquelon. Joseph-Alphonse Desrousseaux, commandant des îles françaises du golfe du Saint-Laurent, au ministre, 8 décembre 1843. BAC, MG1-C11C, vol. 7b, bobine F-505, 2 pages. En 1836, les Anglais fréquentant le secteur de Fortune Bay à Port aux Basques se rendent compte que les échanges entre les habitants de la côte sud et ceux de Saint-Pierre et Miquelon existent pratiquement depuis 1763. Korneski, Conflicted Colony, 25. 
françaises ${ }^{36}$. Sur une note plus positive en termes de sécurité maritime, des rumeurs veulent que la France et l'Angleterre songent à construire des phares sur la côte de Terre-Neuve, entre autres au Chapeau Rouge ${ }^{37}$. En juillet de cette même année, le commandant des îles françaises du golfe du Saint-Laurent, Joseph-Alphonse Desrousseaux, achemine un très long mémoire au ministre. Les sujets dont il traite ne sont pas nouveaux; l'approvisionnement en bois et en appâts, le peuplement anglais des espaces de pêche réservés aux Français sur la côte de Terre-Neuve, le maintien des bonnes relations avec les autorités anglaises et les concessions d'exploitation accordées à des compagnies françaises.

Desrousseaux, tel que sa correspondance l'atteste, a toujours maintenu « d'excellentes relations » avec Sir John Harvey, alors gouverneur anglais de Terre-Neuve ${ }^{38}$. Également capitaine de corvette, Desrousseaux, comme ses prédécesseurs, consacre beaucoup de temps et d'énergie à surveiller les nombreux rapports qu'entretiennent les pêcheurs franco-britanniques dans le cadre de leurs occupations. Il en surgit de nombreux conflits et il ne voit pas de solution pour les régler à court terme, ni même à moyen terme. Deux exemples récurrents de ces fréquentations réciproques résident dans l'approvisionnement de l'archipel en bois de chauffage sur la côte de Terre-Neuve et la quête d'appâts de pêche par les Anglais à Saint-Pierre et Miquelon ou encore par les Français à Terre-Neuve. À cela s'ajoute le peuplement anglais des ports de la côte ouest de Terre-Neuve, pourtant accordés aux pêcheurs français par les décisions ministérielles, entre autres celle du 26 décembre $1826^{39}$. Ces constatations confirment la difficulté qu'éprouvent les diplomates franco-britanniques à élaborer des règles s'appliquant de manière claire dans les colonies. Les réalités locales d'espaces partagés imposent des mesures d'accommodement ne respectant pas toujours l'esprit des traités.

Pour revenir sur la question de la vente d'appâts aux pêcheurs étrangers, quoique défendue par la loi anglaise, elle n'apparait pas avoir été appliquée de manière stricte. Autrement, les pêcheurs français en auraient déjà grandement souffert. C'est peut-être parce que la population anglaise en souffrirait elle-aussi que la règle n'est pas appliquée à la lettre? Les autorités de St. John's ont donc semblé choisir le parti de la «plus nombreuse et la plus misérable de ses sujets », plutôt que d'écouter les plaintes incessantes des marchands anglais ${ }^{40}$. Desrousseaux

36 Non daté. 1844, BAC, MG1-C11C, vol. 7b, 2 pages.

37 Philippe Alfred Guichon de Grand Pont. Saint-Pierre et Miquelon, mars 1844. BAC, MG1C11C, vol. 7b, bobine F-505,1 page. Grandpont est commandant par intérim à Saint-Pierre en 1842 . Saint-Pierre et Miquelon. Liste des commandants. http://www.woridstatesmen.org/Saint-Pierre.html.

38 Joseph-Alphonse Desrousseaux, commandant des îles françaises du golfe du Saint-Laurent, au ministre, 15 juillet 1844. BAC, MG1-C11C, vol. 7b, bobine F-505, 1 page.

39 Mémoire de Desrousseaux, commandant des îles françaises du golfe du Saint-Laurent, sur les actes de son administration, au ministre, 25 juin 1845. BAC, MG1-C11C, vol. 7b, bobine F-505, 8 pages. À noter que les pêcheurs français aussi achetaient parfois des appâts aux Anglais.

40 Cen'est pas tout à fait l'avis de Neary. Il explique que la majeure partie de la boëtte qu'obtiennent les Français provient des pêcheurs anglais résidant sur la côte sud de Terre-Neuve. Et ce, même si depuis 1786, ce genre de commerce est prohibé. En 1845, suite aux démarches du puissant lobby marchand de St. John's, le gouvernement impose une taxe de 3 shillings « per hundred-weight » sur 
attribue ce contexte de tolérance des autorités anglaises à la volonté de compromis des deux gouvernements, à Saint-Pierre et à St. John's. Lui-même s'est toujours fait un point d'honneur de maintenir avec la capitale terre-neuvienne « ce caractère de franchise, de sincérité et de bienveillance mutuelle, qui seul peut assurer à notre établissement la jouissance absolue d'avantages aussi précieux ${ }^{41}$. Par ses propos, Desrousseaux sous-entend que l'esprit de bon voisinage repose en bonne partie sur l'ouverture d'esprit des autorités des deux nations. Cependant, un changement de personnel peut faire basculer ces relations dans la rigidité et la mésentente. Il se permet de citer deux exemples confirmant ses habiletés diplomatiques : sa réclamation en faveur de la veuve Baudouard, dont le mari fut tué sur les fonds de pêche anglais en juillet $1843^{42}$ et la remise, sans frais, de trois goélettes françaises saisies dans la baie du Désespoir à l'automne $1843^{43}$. Ce genre d'accomplissement lui fait dire qu'il « laisse à son successeur les choses dans un état très satisfaisant ».

Laisser les choses dans un état satisfaisant doit ici se comprendre dans ce contexte de compromis permettant de maintenir le calme tout en faisant une application plutôt discrète des traités. Il y a donc lieu de percevoir ici l'écart existant entre la compréhension et la détermination de faire respecter les clauses des traités par le biais des officiers sur le terrain. La question que l'on doit se poser est de savoir si cette position est jugée véritablement réaliste par les diplomates ou si euxaussi savent fort bien que la clé du succès diplomatique réside dans l'habilité des officiers de frégate à faire usage de jugement et de parcimonie.

En ce qui a trait au peuplement anglais de la côte terre-neuvienne où les Français peuvent pêcher, Desrousseaux estime que la solution à cette problématique proviendra de discussions diplomatiques entre les deux pays. Ce qui sous-entend que dans l'optique des officiers de Marine, l'application des clauses des traités n'assurent nullement le maintien de la paix et le respect des règles par les pêcheurs et les habitants des espaces de côtes contestées. Il pense surtout à la baie de SaintGeorges et à Cod Roy en général. La fréquentation entre pêcheurs des deux nations qui en résulte, ne peut que faciliter la contrebande de morue anglaise ${ }^{44}$ et fausser

l'exportation de la boëtte. De cette manière, ce serait plutôt les pêcheurs de Terre-Neuve qui iraient vendre la boëtte à Saint-Pierre. Neary, 98 .

41 Mémoire de Desrousseaux, 26 juin 1845, 3.

42 Il s'agit plus précisément de la pirogue $n^{\circ} 39$, montée par un marin et deux mousses. Beaudouard aurait été abattu par l'officier Butler, alors dans un canot détaché du navire de guerre anglais Electra. L'embarcation française aurait alors été surprise à pêcher sur les fonds anglais. Dans la foulée de cet événement, la goélette d'État la Gentille dépose une plainte formelle aux autorités britanniques à St. John's. Émile Sasco et Joseph Lehuenen, Chronologie des îles Saint-Pierre et Miquelon, 1998, disponible en ligne. 18 juillet 1843, 25. Le mois suivant, une corvette de guerre anglaise vient à Saint-Pierre pour exprimer les regrets officiels des autorités britanniques qui attribuent l'incident « au hasard et à l'imprudence » et «non à l'intention ». Sasco et Lehuenen, Chronologie, 7 août 1843, 25.

43 Mémoire de Desrousseaux, 26 juin 1845, 4. Il s'agit peut-être de celles surprises à y couper du bois de chauffage en novembre 1843. Sasco et Lehuenen, Chronologie, $1^{\text {er }}$ novembre 1843, 25.

44 En 1831 émanent des plaintes contre des dommages infligés par des pêcheurs français à un établissement de pêche appartenant à Richard Perry à Placentia. Mais ce serait plutôt l'œuvre de contrebandiers d'alcool que de pêcheurs. En échange de brandy, ces derniers acceptent la morue 
les critères du système de prime des pêcheries françaises. Mais l'administration française de Saint-Pierre n'a aucune manière de l'empêcher ${ }^{45}$. Toutefois, Desrousseaux prétend avoir trouvé un moyen détourné de freiner l'augmentation de la population anglaise, soit en renouvelant la concession des «pointes » de côtes sous autorité française à la compagnie Campion et Théoroulde en juin 1842. Quoique révoquée par le ministère en 1843, Desrousseaux la renouvelle de sa propre initiative à titre provisoire, jusqu'à la « conclusion des conférences entamées avec le gouvernement anglais $»^{46}$.

Toujours au fait des rumeurs provenant du front diplomatique, les officiers en poste à Saint-Pierre s'inquiètent de leur impact sur les droits de pêche français à Terre-Neuve. Par exemple, en 1846, le commandant de Saint-Pierre, Joseph Marie Fidèle Délécluse, rapporte certaines rumeurs voulant que la France envisage de céder Saint-Georges contre Belle Isle. Pareille décision risque de nuire grandement aux intérêts des armateurs de Saint-Pierre au profit d'une industrie des pêcheries françaises déjà riche. Doit-on en conclure qu'une telle transaction diplomatique résulte du lobbying de grandes entreprises métropolitaines françaises ${ }^{47}$ ? La même année, un rapport de Gaulin, commandant la goélette gouvernementale la Gentille, tente d'expliquer certains changements dans les lieux exploités traditionnellement par les goélettes de l'archipel ${ }^{48}$.

Ces lieux de pêche incluent les localités de Port-au-Port, Cod Roy et baie SaintGeorges. Gaulin, lui, tente d'abord de démontrer pourquoi les pêcheurs de SaintPierre et Miquelon ne fréquentent presque plus certains havres de Terre-Neuve, pourtant sous juridiction française. En premier lieu, il semble y avoir absence de morue et de caplan à Port-au-Port où il n'y a plus que des pêcheurs de SaintMalo et des îles Jersey et Guernesey. Ils y pêchent surtout le hareng. Autrefois, les goélettes de l'archipel y débutaient leur pêche avant de se diriger vers les îles de la Madeleine. Mais maintenant, elles préfèrent mettre le cap directement sur les bancs côtiers de l'archipel, pour ensuite se rendre sur le Grand Banc ${ }^{49}$.

En deuxième lieu, Gaulin replonge dans le passé pour expliquer que le processus de peuplement de Saint-Georges remonte à 1817. Y arrive alors un dénommé

anglaise. Il s'agit là d'une violation des traités qui permettent aux Français de vendre de la morue anglaise à moindre prix sur les marchés européens. Ces accusations sont appuyées par Joseph Tucker, résident de Little Placentia, qui cible plus précisément des pêcheurs de Saint-Pierre et Miquelon. Richard Perry (Poole) à Lord Viscount Goderich (F.J. Robinson), 24 février 1831. CO 194, vol. 83, bobine B-534, 298-298v. Voir aussi pages 299, 21 février 1831, lettre de Joseph Tucker.

45 Mémoire de Desrousseaux, 26 juin 1845, 5.

46 Mémoire de Desrousseaux, 26 juin 1845, 7. Dans la même veine et pour les mêmes raisons, il accorde aussi une concession de cinq ans (jusqu'en 1848) à la compagnie Harvins de Saint-Malo à Port au Port sur la côte de Terre-Neuve.

47 Extrait d'un rapport annuel du commandant de Saint-Pierre et Miquelon, 31 août 1846. BAC, MG1-C11C, vol. 7b, bobine F-505, 2 pages.

48 Rapport adressé au commandant des îles Saint-Pierre et Miquelon sur les pêcheries opposées à ces îles à la côte ouest de Terre-Neuve. Par monsieur Gaulin, commandant de la goélette la Gentille, $1^{\text {er }}$ octobre 1846. BAC, MG1-C11C, vol. 7b, bobine F-505, 17 pages.

49 Rapport de Gaulin, ${ }^{\text {er }}$ octobre 1846, 1, 5-6. 
Benoist, marié à une amérindienne, accompagné des Denys, des Penny, des Stuart et des Museroy. Tel que mentionné auparavant, il y a donc dès lors cohabitation multiethnique et ces gens y pêchent surtout le saumon. Sir Charles Hamilton, administrateur anglais, par une proclamation datant de 1822, tente de les chasser mais sans succès et depuis, rien n'a été fait pour pallier ce problème. Saint-Georges semble être aussi devenu un refuge pour les marins déserteurs et des Acadiens en provenance du Cap-Breton et du Nouveau-Brunswick. Ces derniers n'y pêchent que l'anguille et les Anglais le hareng; il n'y a plus de morue dans la baie. C'est ce qui explique pourquoi les habitants de l'archipel n'y vont plus ${ }^{50}$.

Cependant, Gaulin ne comprend pas pourquoi les goélettes de l'archipel ne fréquentent pas plus Cod Roy, encore «un excellent lieu de pêche $»^{51}$. Ceci étant dit, il demeure persuadé qu'il faille maintenir la juridiction française sur SaintGeorges. Surtout depuis que le gouvernement britannique impose une taxe (droit de douane?) sur le hareng et le caplan exportés à Saint-Pierre. Si jamais cet approvisionnement vient à manquer, Saint-Georges devient une alternative pour « l'approvisionnement en boëtte » des navires banquiers de la métropole (France) ${ }^{52}$.

Selon l'officier de Marine Jules Sandeau, il y a bien des négociations en 1844 pour permettre aux pêcheurs des deux nations de travailler librement et d'éviter les accrochages. Mais, comme lors des pourparlers de 1851, elles se soldent par une impasse. Globalement, la France tente constamment de préciser « la nature de ses droits sur les pointes les plus fréquentées » par ses pêcheurs, « en échange d'une concession de droit de pêche commune dans les baies qu'elle fréquente peu en raison de la diminution de ses armements ». La baie de Saint-Georges demeure donc un enjeu important ${ }^{53}$.

\section{Une harmonie durable sur les côtes? 1849-1864}

Entre 1848 et 1851, autant sur la scène diplomatique en métropole qu'entre SaintPierre et St-John's, les relations franco-britanniques semblent plutôt harmonieuses. Ainsi, en 1848, la corvette de guerre anglaise Arraché visite 1'archipel et « la meilleure entente existe entre les autorités françaises et anglaises ${ }^{54}$. Même

50 Rapport de Gaulin, $1^{\text {er }}$ octobre 1846, 1. Des commentaires peu flatteurs sont exprimés à l'égard des Acadiens dans un rapport de 1847 par le lieutenant Gautier. « Les Acadiens d'origine française, mais presqu'abandonnés à eux-mêmes sans loi ni foi depuis la domination britannique, forment une population tout à fait à part, population infime et abâtardie, vivant au jour le jour, et ne se distinguant de leur voisin les Anglais que par leur inertie et leur oisiveté ». Plus loin, ce même auteur écrit que «quelques Acadiens moins paresseux sont employés par les Anglais comme manœuvres ». 18 juin 1847. Rapport adressé à Monsieur le Commandant des îles Saint-Pierre et Miquelon sur les pêcheries effectuées à ces îles et à la côte de Terre-Neuve. Par M. Gautier, Lieutenant de vaisseau, commandant de la goélette d'État la Gentille. Dans BAC.MG1-C11 ${ }^{\mathrm{C}}$, bobine F-505.

51 Rapport de Gaulin, $1^{\text {er }}$ octobre $1846,2$.

52 Rapport de Gaulin, 1er octobre 1846, 14.

53 Jules Sandeau, «Pêcheries de Terre-Neuve et les traités », dans Rompkey, Les Français à Terre-Neuve...op.cit., 279.

54 Paris. Au ministre, 8 octobre 1848. BAC, MG1-C11C, vol. 7b, bobine F-505, 1 page. 
constat en 1851, lorsque la frégate britannique Alarm fait relâche à Saint-Pierre. Son maître, Ramsay, n'est nullement hostile envers les pêcheurs français dans le cadre de ses fonctions de surveillance. Il est même d'avis que Londres permettrait aux Français de l'archipel « d'importer leurs appâts des côtes terre-neuviennes anglaises, quoiqu'en disent les commerçants de Saint-Jean (St. John's) $»^{55}$. Selon Korneski, à partir de 1850 sur la côte sud, les Français sont les bienvenus en autant qu'ils maintiennent leur commerce avec les habitants en leur achetant de la boëtte et du hareng ${ }^{56}$. Mais la situation semble moins calme le long des côtes des provinces maritimes à l'été 1852. Incidemment, selon Blake Earl, la forte présence des frégates britanniques vise alors à forcer les Américains à accepter le libreéchange (entente de 1854) avec les colonies anglaises d'Amérique du Nord ${ }^{57}$.

$\mathrm{Au}$ moment de l'implantation du gouvernement responsable en 1855, contrairement au climat optimiste menant les deux empires vers l'entente cordiale et l'alliance de la Guerre de Crimée, St. John's estime que les pêcheries françaises représentent un danger important pour le commerce international de la colonie. Mais Neary pense plutôt que les marchands locaux exagèrent cet inconvénient que représente alors la compétition française, utilisant ce motif pour masquer leur propre incapacité 58 . Rappelons que c'est aussi l'époque de la Convention francobritannique, signée le 14 janvier 1857, relative aux droits de pêche à Terre-Neuve ${ }^{59}$. D'ailleurs, la législature de Terre-Neuve s'affirme encore davantage en février de la même année, alors qu'elle rejette la proposition d'une convention francobritannique sur les pêcheries ${ }^{60}$. D'un même élan, le Conseil législatif demande au gouverneur de solliciter l'intervention de l'amiral britannique de la Station navale d'Amérique du Nord et des Antilles, pour assurer la protection des pêcheries côtières et prévenir les sources de friction entre pêcheurs franco-britanniques ${ }^{61}$.

En dépit de ces relations plutôt harmonieuses, la France semble encore en réflexion quant au choix à faire dans la région. Faut-il céder Saint-Georges ou l'accès au golfe du Saint-Laurent? On semble croire qu'en cédant Saint-Georges, les pêcheurs de l'archipel pourraient plus facilement « exporter leur morue aux

55 Saint-Pierre et Miquelon. Commandant de Saint-Pierre au ministre, 13 juin 1851. BAC, MG1C11C, vol. F-505, 2 pages. Il est fort probable que ce sujet est abordé en mai 1856, lors de la visite de la corvette à vapeur Argus à Saint-Pierre. Sasco et Lehuenen, Chronologie, 16 mai 1856, 31.

56 Korneski, Conflicted Colony, 29-30.

57 Earl, 4.

58 Neary, 96.

59 Pour replonger au cœur de cette affaire, voir 14 janvier 1857. Convention anglo-française relative aux pêcheries de Terre-Neuve. https://books.google.ca

60 T. Hammond à Herman Merivale, 11 mai 1857. CO 194, vol. 151, bobine B-674, 520-521. L'on sait que le gouverneur de Terre-Neuve lui-même, Sir Darling, se rend à Saint-Pierre en août 1856. Il y est accueilli en grande pompe avec dîner et soirée dansante. Sasco et Lehuenen, Chronologie, 6 août 1856, 31. Également, l'année suivante, le gouverneur Darling assure le commandant Gervais que les auteurs du pillage de la goélette française Marie Élisabeth ont été « sévèrement réprimés ». Sasco et Lehuenen, Chronologie, 28 février 1857, 32.

61 J. Kent, 21 mai 1858. CO 194, vol. 152, bobine B-698, 510-511v. 
États-Unis, au Canada et en Nouvelle-Écosse ${ }^{62}$.

De concert avec la volonté franco-britannique de cimenter leur processus d'alliance, en juin 1857, l'Atalanta, navire monté par 130 hommes et affecté à la station anglaise de Terre-Neuve, arrive à Saint-Pierre sous le commandement de l'officier Pasley. À cette occasion, les deux administrations réitèrent leurs mandants respectifs à double volet; protéger les pêcheurs de chaque nation, tout en leur imposant le respect des traités. Par marque de respect, le navire anglais a "salué le pavillon français de 21 coups de canon », ce qui lui a été rendu par « l'artillerie de la place ». La capitaine de port de Saint-Pierre, à titre de délégué du commandant français, présente les « compliments et mes offres de service à monsieur le commandant Pasley ». Ce jour-là et le lendemain, les deux commandants s'échangent des visites de courtoisie, souhaitant réciproquement « que le meilleur accord continuerait de régner entre les divisons navales ». Pasley accepte finalement de « diner à l'hôtel du gouvernement » en compagnie de JeanFrançois Gervais et des « principaux fonctionnaires de la colonie $»^{63}$.

Finalement, à la veille de la Confédération canadienne, en octobre 1864, la visite du vapeur anglais le Lys à Saint-Pierre confirme la continuité des bonnes relations entre les administrateurs des stations anglaise de Terre-Neuve et française de SaintPierre. Il est toutefois important de ne pas oublier la présence du troisième joueur dans la problématique des droits de pêche : les pêcheurs américains. En effet, on note la présence du commissaire des pêcheries de l'Amérique du Nord britannique, Howe, chargé par le gouvernement britannique de déterminer de concert avec un commissaire des États-Unis les parties du littoral de Terre-Neuve et du Labrador où les Américains sont « autorisés aux termes des traités, à se livrer à la pêche ${ }^{64}$. À cette occasion, Howe confirme la «bonne entente » qui règne sur tous les points des côtes est et ouest de Terre-Neuve entre les nationaux franco-britanniques. Le commandant Orcy maintient que durant le séjour de trois jours de ce navire anglais à Saint-Pierre, l'État-major anglais a fait l'objet d'attention et de courtoisie de la part des fonctionnaires coloniaux français.

\section{Conclusion}

La situation historiquement sensible des droits de pêche partagés à Terre-

62 Saint-Pierre et Miquelon, 12 juin 1854. BAC, MG1-C11C, vol. 7b, bobine F-505, 1 page.

63 Saint-Pierre. Gervais, commandant de Saint-Pierre et Miquelon, à Gamelin, ministre, 17 juin 1857. BAC. MG1-C11C, vol. 7b, bobine F-505, 3 pages.

64 Saint-Pierre. Orey, commandant de Saint-Pierre et Miquelon. Copie de sa missive précipitée, 3 novembre 1864. BAC, MG1-C11C, vol. 7b, bobine F-505, 2 pages. Il n'est pas certain s'il s'agit d'Orey ou d'Orcy. Son prénom pourrait être Charles-Faure. Dans ses travaux, Korneski fait référence à la dispute de 1878 entre les résidents de Fortune Bay et des goélettes américaines de Gloucester (Massachusetts). Même si ces dernières ont le droit de pêcher près des côtes en vertu des clauses du Traité de Washington de 1871, ils n'en violent pas moins les règles imposées par le code de conduite non écrit. Code que doivent s'imposer les étrangers pénétrant dans les eaux côtières. Par exemple, ils ne doivent pas pêcher eux-mêmes la boëtte mais plutôt l'acheter des pêcheurs locaux. Korneski, Conflicted Colony, 16. 
Neuve doit donc s'inscrire dans une dynamique internationale des intérêts francobritanniques. L'on a pu constater que ces intérêts sont tantôt communs et tantôt opposés. Ainsi, tout en cheminant vers l'entente cordiale et l'alliance lors de la Guerre de Crimée, les deux puissances s'observent mutuellement lorsque vient le temps d'affirmer leurs ambitions coloniales respectives. C'est essentiellement le même scénario qui prévaut sur les côtes de Terre-Neuve, dans le cadre des missions de surveillance des officiers de frégates franco-britanniques. Mais la situation est moins calme dans les activités britanniques de surveillance le long de côtes des provinces maritimes. Il semble bien que les intervenions des frégates britanniques y résultent bien plus souvent en arrestations formelles qu'à Terre-Neuve.

À la lecture de cette abondante correspondance entourant les tentatives franco-britanniques de faire respecter les traités et de maintenir l'ordre sur les zones françaises de la côte terre-neuvienne, il est intéressant de noter que l'autre enjeu consiste à limiter la croissance de la population anglaise sur ces mêmes territoires. Mais le chercheur doit en retirer d'autres conclusions fortes intéressantes. D'abord, les commandants franco-britanniques des stations navales de Terre-Neuve ont grandement intérêt à collaborer pour maintenir l'ordre. Ainsi, en examinant le ton des propos des deux camps, il devient évident qu'avec le temps, les deux parties constatent qu'ils ne sont pas en mesure d'empêcher à la fois le peuplement anglais des côtes sous juridiction française ou encore d'exclure complètement les pêcheurs des deux nations de pêcher dans les zones opposées. Il y a également lieu de se demander si l'alternance d'accalmie et de turbulence n'est pas également attribuable aux rendements des pêcheries. Par exemple, s'il y a surpêche dans certaines localités du territoire à l'étude, est-ce que cela incite les pêcheurs à enfreindre les clauses des traités? Ou, au contraire, en temps de très bonnes saisons, est-ce que les autorités franco-britanniques ont tendance à relâcher la surveillance? Il y a donc matière à penser qu'en contexte de rareté du poisson, le stress découlant d'une subsistance plus difficile puisse provoquer des écarts de conduite.

Donc, au fur et à mesure que s'écoulent les années, émerge une certaine solidarité entre les officiers patrouilleurs et les fonctionnaires franco-britanniques. Il est cependant difficile de déterminer à quel point un phénomène comparable puisse exister chez les pêcheurs des deux nations. S'il y a complicité entre les Français de l'archipel et les Anglais du French Shore, ce serait peut-être au détriment des pêcheurs métropolitains des deux pays. À cela s'ajoute la dynamique du respect du fameux code de comportement abordé lors de note bilan historiographique. Code auquel doivent s'astreindre les pêcheurs de toutes les nations. Par exemple, ils doivent acheter leur boëtte des pêcheurs et des habitants de la côte sud de TerreNeuve et non la pêcher eux-mêmes.

Il est donc permis de penser que les pêcheurs coloniaux, au même titre que les politiciens coloniaux terre-neuviens, sont à développer une solidarité régionale allant au-delà de leurs origines ethniques respectives. En fait, il y a lieu de situer leur comportement dans les réflexions suscitées par l'ouvrage classique de Rediker et Linebaugh, à savoir que ces pêcheurs réagissent de manière comparable à celle 
d'autres catégories de main-d'œuvre maritime par « leur résistance » et leur volonté de « développer un projet social égalitaire ». Ils s’inscrivent ainsi, peutêtre sans s'en rendre compte, dans des « réseaux atlantiques », dans un « univers multiethnique maritime, fait de résistance et de solidarité ${ }^{65}$.

65 Sébastien Lecompte-Ducharme, « Une histoire atlantique par le bas, ou une histoire mondiale globale tout court? L'hydre aux mille têtes de Marcus Rediker et Peter Linebaugh », Histoire globale. Le blog, 19-9-2016. Publié par Laurent Testot. http://blogs.histoireglobale.com/une-histoireatlantique. 